\title{
Hippocampal lesions and immobility responses in the rat
}

\author{
MICHAEL L. WOODRUFF and SUZANNE D. BAILEY \\ Middlebury College, Middlebury, Vermont 05753
}

\begin{abstract}
The present study was conducted to compare the effects on several immobility reactions of hippocampal ablation in rats. Hippocampal lesions were found to increase significantly the duration of restraint-induced immobility, but these same lesions significantly increased movement in response to footshock and in an open field, indicating a reduction in the tendency for rats with hippocampal lesions to become immobile in these situations. These results are interpreted as indicating that hippocampal lesions in animals do not inevitably lead to a decreased tendency to become immobile in stressful situations and that the hippocampus in the adult rat inhibits behaviors which immature rats demonstrate more readily than mature rats.
\end{abstract}

Several authors have concluded that a reduction in the ability to become immobile is a central behavioral effect produced by hippocampal lesions (Altman, Brunner, \& Bayer, 1973; Blanchard, Blanchard, Lee, \& Fukunaga, 1977; Isaacson, 1974). As Isaacson (1974, p. 161) has noted, the reduced tendency of animals with hippocampal lesions to become immobile is most likely to be observed "under conditions of fear or stress." For example, while rats with hippocampal lesions exhibit as many episodes of immobility in response to footshock as control animals, these episodes are of significantly shorter duration than those of controls (Blanchard et al., 1977). Rats with hippocampal lesions are also less likely than controls to become immobile in the presence of a predator (Blanchard \& Blanchard, 1972; Kim, Kim, Kim, Kim, Chang, Kim, \& Lee, 1971), or in response to a conditioned stimulus which has been associated with electric shock (Blanchard \& Fial, 1968).

Immobility can also be produced in members of many species by sudden physical restraint and continued pressure on body parts. Immobility induced in this manner continues after restraint is removed and has been referred to as "death feigning," "animal hypnosis," "the immobility reflex," or "tonic immobility." During this form of immobility, the animal exhibits a reduction in reactivity to external stimulation, loss of the righting reflex, and depression of polysynaptic reflexes (Carli, 1977). Ratner (1967) has proposed that immobility of this sort, which is caused by close contact with a predator,

Portions of this paper were presented at the 86th annual convention of the American Psychological Association. Please address reprint requests to Michael L. Woodruff, PhD, who is now with the Department of Anatomy, College of Medicine, East Tennessee State University, P.O. Box 19960A, Johnson City, Tennessee 37601. is the final stage in a series of four, distancedependent, defensive patterns. Upon observing the predator at a distance, the prey freezes, but flees if the predator approaches. If caught, the prey fights, but becomes immobile if its struggles are unsuccessful. This form of immobility would seem, therefore, to occur in a condition of stress. Moreover, Gallup (1974) has presented a convincing argument that a fear state is an important variable in the modulation of tonic immobility.

If reduction of the tendency to become immobile in conditions of fear and stress is a principal consequence of hippocampal ablation in infrahuman animals, then it would be predicted that hippocampal lesions would attenuate immobility produced by sudden restraint and pressure on the body. However, the results of an experiment by Woodruff, Hatton, and Meyer (1975) indicate that hippocampal ablation in rabbits significantly potentiates immobility induced in this way. The results of this experiment indicate that there are limits to the hypothesis that hippocampal destruction leads to decreased immobility. Most of the data supporting this hypothesis have been gathered from rats and it may be that species differences exist in the behavioral response to hippocampal destruction. However, hippocampal ablation in either rabbits or rats facilitates the acquisition of two-way active avoidance and impairs the acquisition of passive avoidance (Isaacson, Douglas, \& Moore, 1961; Isaacson \& Wickelgren, 1962; Papsdorf \& Woodruff, 1970). These observations suggest that hippocampal damage produces similar behavioral effects in rabbits and rats. It may also be, then, that the precise nature of both the stimuli which induce immobility and the particular immobility response under study determines whether hippocampal destruction will either attenuate or potentiate immobility. 
The present study was conducted to determine whether rats given hippocampal lesions would exhibit increased restraint-induced immobility as compared to controls. Activity levels in response to footshock and in an open field were also measured. These latter two experiments were conducted to determine whether the lesions produced in the rats used in this study would result in the replication of effects obtained in previous experiments in other laboratories. As indicated above, rats with hippocampal damage usually exhibit less immobility than controls in these situations (e.g., Blanchard et al., 1977). Finally, three rats were prepared using the Fink-Heimer method for staining degenerating axons and terminals. This was done to verify that the lesions given the rats used in the behavioral paradigms produced degenerative changes similar to those typically associated with lesions of the hippocampal formation (Siegel, Edinger, \& Ohgami, 1974; Valenstein \& Nauta, 1959).

\section{METHOD}

\section{Subjects}

Twenty-seven male hooded rats obtained from Canadian Breeding Farms, St. Constant, Quebec, were used. The rats weighed between 280 and $330 \mathrm{~g}$ at the time of surgery and were housed individually. Food and water were available ad lib throughout the experiment. A 12:12 light-dark cycle was in effect for the colony room. All testing was done between the 5th and the 9th hour of the light period. Eight rats received bilateral electrolytic lesions of the hippocampus (Group $\mathrm{H}$ ); eight rats served as sham-operated controls (Group S); and eight rats were placed in the normal control group (Group N). Three rats were given unilateral hippocampal lesions. The brains of these rats were processed for analysis of lesion-induced degeneration patterns.

\section{Operative Procedure}

The rats were placed at random into one of the three groups. Each rat in Groups $\mathrm{H}$ and $\mathrm{S}$ was anesthetized with sodium pentobarbital (Nembutal, $60 \mathrm{mg} / \mathrm{kg}$, IP). The head was shaved and the animal was positioned in a Kopf stereotaxic instrument. A midline incision was made, and the scalp, muscles, and fascia were retracted to expose the dorsum of the skull. After three small holes had been drilled through the skull, electrodes made from 00-ga stainless steel insect pins, insulated with Formvar except for approximately $.5 \mathrm{~mm}$ at the tip, were lowered and anodal electrolytic lesions were produced in the rats in Group H. An anal cathode served as a reference. The stereotaxic coordinates (Pellegrino \& Cushman, 1967) for the rats in Group H were anterior-posterior $-1.8,-3.2$, and -4.4 with bregma as the reference. The corresponding lateral placements were $\pm 1.8, \pm 5.3$, and \pm 5.2 . Dura served as the reference for the ventral coordinates, which were -3.1, -5.3, and -5.0. A 2.0-mA direct current was used for each placement. For the most anterior placement, the current was applied for $12 \mathrm{sec}$. For the other placements, the current remained on for $15 \mathrm{sec}$. The sham-operated animals received the same treatment as the experimental rats, except that the current was not turned on. Upon completion of the operation, each rat received 30,000 units of penicillin (Flocillin) to combat possible postoperative infections. A 21-day recovery period was allowed before behavioral testing was begun.

\section{Behavioral Testing}

Twenty-two days after surgery, the rats were assessed for restraint-induced immobility. Each rat was carried to the test room in its home cage. The rat was then removed from its cage and placed upright in a V-shaped trough. The trough was constructed from 9-mm-thick unpainted plywood. The sides were $23.6 \mathrm{~cm}$ high, $47.6 \mathrm{~cm}$ long, and joined at a right angle. A 100-W surgical lamp was positioned $2.0 \mathrm{~m}$ above the trough and was focused on it. This lamp provided the room's only illumination.

Thirty seconds after it was placed in the trough, the experimenter grasped the rat around the thorax and inverted it into the trough. Pressure was maintained on the rat's thorax for $60 \mathrm{sec}$, at which time the hand was removed gradually. If the rat remained inverted, a stopwatch was started. The watch was stopped when the rat righted itself. The duration of immobility in the inverted position was the dependent variable. After the rat righted itself, it was kept in the trough for $30 \mathrm{sec}$. It was then inverted and restrained again. If the rat did not remain immobile when the hand was removed, it was kept in the trough for $30 \mathrm{sec}$ and the procedure was then repeated. A total of five trials was given each rat in this manner, after which it was returned to the colony room.

An automated shuttlebox (BRS/LVE Model RSC-044) was used to assess the level of activity of the rats in response to a .4-mA scrambled footshock produced by a Grason-Stadler shock generator (Model E1064GS). The number of times the rat crossed the center of the shuttlebox was the dependent variable. Presentation of the shock and recording of crossings was controlled by electromechanical relays, timers, and counters located in a room adjacent to the test room. The shuttlebox was illuminated by a $40-\mathrm{W}$ lamp positioned $1.5 \mathrm{~m}$ above its top. Each rat was tested in this apparatus for 3 consecutive days beginning on Postoperative Day 28. The rat was first placed in the shuttlebox for $5 \mathrm{~min}$ without being shocked, and the number of crossings from one chamber to the other was recorded. The .4-mA shock was then introduced through the grid floor of the shuttlebox for $1 \mathrm{~min}$, and the number of crossings was recorded. The rat remained in the shuttlebox for an additional $5 \mathrm{~min}$, and the number of crossings was recorded. This procedure was repeated on the following 2 days.

Each rat was tested for activity in a larger area on Postoperative Days 36, 37, and 38. The area was a square plywood box painted flat gray. It measured $90 \mathrm{~cm}$ on a side and was divided into 36 equal area squares by black lines painted on its floor. The sides of the box were $35 \mathrm{~cm}$ high. A $60-\mathrm{W}$ lamp, positioned $1.5 \mathrm{~m}$ above the center of the floor of the box, served as the sole source of illumination in the room. The duration of each test period was $10 \mathrm{~min}$. The experimenter sat approximately $1.0 \mathrm{~m}$ from one edge of the box. The number of lines the rat crossed with all four feet was recorded, as was the number of times the animal reared up and the number of fecal pellets deposited during the test period. The area was cleaned thoroughly after each rat had completed its test.

\section{Histology}

After behavioral testing had been completed, each rat in both Group $\mathrm{H}$ and Group S was given an overdose of Nembutal and intracardially perfused with $.9 \%$ saline and $10 \%$ Formalin. The brain was postfixed in $10 \%$ Formalin for 30 days and then embedded in celloidin. Coronal sections were cut at $30 \mu \mathrm{m}$. Every seventh section from the anterior septal area through the hippocampus was slide mounted and stained with thionin. Every eighth section was stained using the Weil method for myelin sheaths (Clark, 1973) and then mounted on a microscope slide. The three rats that had received unilateral hippocampal lesions were overdosed and perfused 4 days after the lesion. The brains were postfixed for from 45 to 55 days. Frozen sections were cut at $30 \mu \mathrm{m}$ and every fifth section was stained for degenerating axons and terminals (Wiitanen, 1969).

\section{RESULTS}

\section{Histology}

Inspection of the thionin- and Weil-stained sections revealed that a substantial portion of the anterior- 

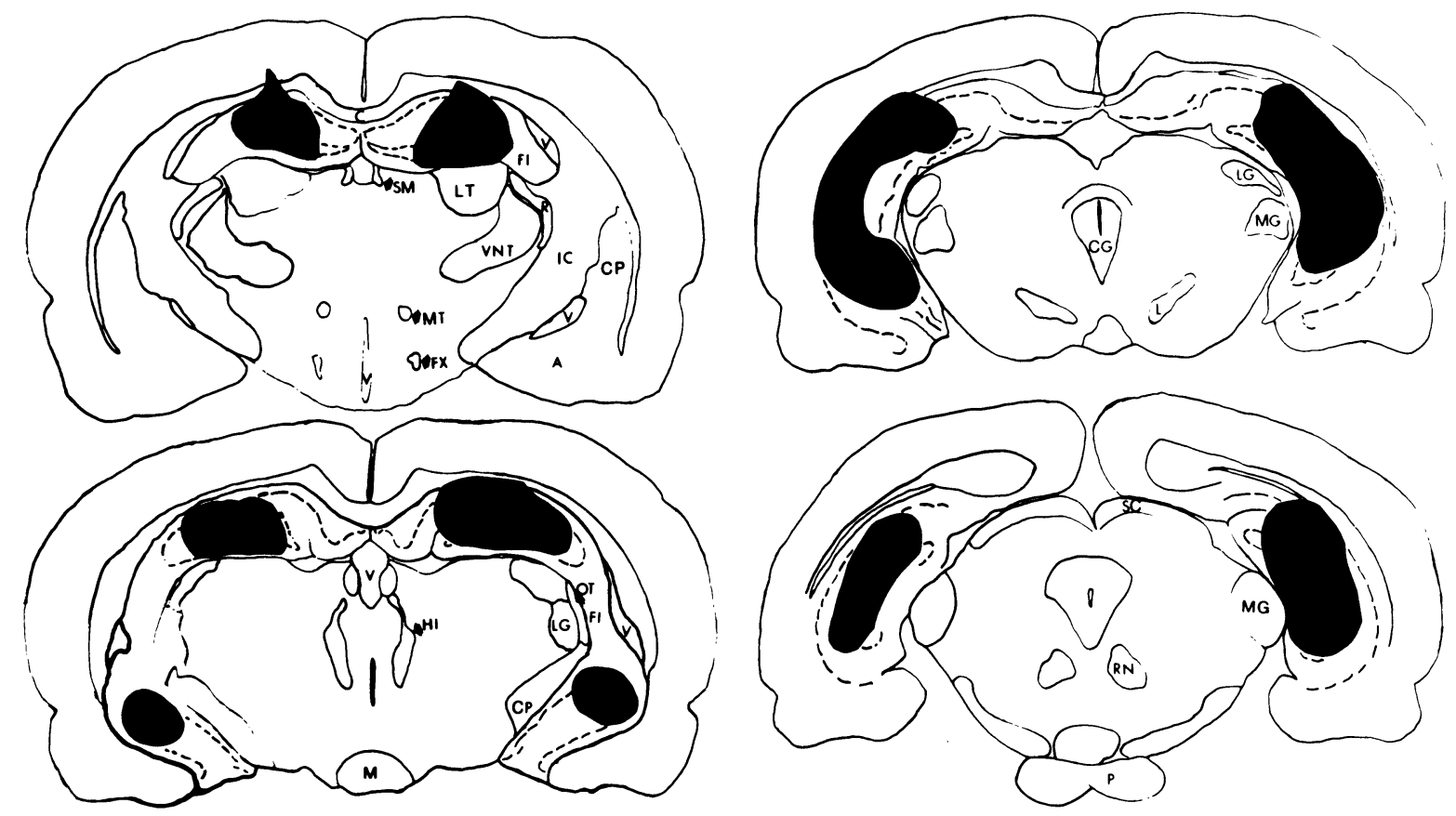

Figure 1. Projection drawings of four levels of a representative hippocampal lesion. Abbreviations: A, amygdala; C, corpus callosum; CG, central gray; CP caudate-putamen; Cp, cerebral penduncle; FI, fimbria; FX, fornix; HI, habenulo-interpenduncular tract; IC, internal capsule; L, lemniscus; LG, lateral geniculate nucleus; LT, lateral thalamic nucleus; M, mammillothalamic tract; OT, op tic tract; P, pons; RN, red nucleus; SC, superior colliculus; SM, stria medullaris; V, ventricle; VNT, ventral thalamic nucleus.

dorsal portion of the hippocampus had been destroyed by the lesion. The hippocampus located above the stria medullaris remained intact in all eight rats. The anterior hippocampus located above the lateral nucleus of the thalamus was destroyed in all eight rats, and destruction extended laterally to that part of the structure located adjacent to the medial edge of the dorsal lateral geniculate nucleus. Unilateral damage to the dorsal quarter of the lateral thalamic nucleus had occurred in three rats, but this structure did not sustain bilateral damage in any rat. Only slight damage was done to the anterior part of the ventral hippocampus. As can be seen from Figure 1, the more posterior portions of both the dorsal and ventral hippocampus sustained substantial damage. The medial geniculate nucleus had been damaged unilaterally in two rats, but no rat showed bilateral damage to this structure. The corpus callosum had been damaged bilaterally to some extent in every rat in Group H. The subicular region had also been damaged in each rat in Group $\mathrm{H}$, but in only three rats was there observable damage to the entorhinal cortex.

The rats in Group S had all sustained damage to the neocortex and corpus callosum. The electrode tracks had penetrated the hippocampus. Destruction to this structure was limited to the electrode track itself in these animals. The damage to the neocortex was comparable to that for Group $\mathrm{H}$, however, rats in Group $\mathrm{H}$ suffered more damage to the corpus callosum.
The lesions produced in the rats prepared for the Fink-Heimer method were similar in extent to the lesions produced in the rats in Group $\mathrm{H}$, except, of course, that they were unilateral. Microscopic analysis of these preparations indicated that the lesions produced for these experiments caused degenerative patterns which were virtually identical to those previously reported. Very heavy degeneration was observed throughout the course of the fornix on the side of the lesion, while the contralateral postcommissural fornix showed little, if any, evidence of degeneration. Degeneration was observed in both the medial and lateral septal nuclei, as well as nucleus accumbens, the nucleus of the diagonal band, and the olfactory tubercle. Degeneration was also observed in the contralateral hippocampus.

Some degeneration was followed from the fornix into the corpus callosum, probably as a component of the dorsal fornix, but could not be followed further because of degeneration caused by damage to the callosum itself. A slight amount of degeneration was observed to leave the fornix column and was followed to the periventricular zone of the anterior hypothalamus by way of the cortico-hypothalamic tract. Other areas in which degeneration was found included the lateral preoptic area, the lateral hypothalamus, and the lateral part of the medial mammillary nucleus. Sparse degeneration was observed in the anteroventral nucleus of the thalamus, but little, if any, was observed in the anterodorsal nucleus. 
Table 1

Median and Range (in Seconds) for the Duration of Restraint-Induced Immobility for Each Group

\begin{tabular}{lcc}
\hline & Median & Range \\
\hline Group H & 39.0 & $0-175$ \\
Group N & 3.5 & $0-45$ \\
Group S & 2.5 & $0-12$ \\
\hline
\end{tabular}

Table 2

Mean Number of Shuttlebox Center Crossings for Each Group in Response to 1-Min Footshock

\begin{tabular}{lccc}
\hline & Day 1 & Day 2 & Day 3 \\
\hline Group H & 7.37 & 3.50 & 3.50 \\
Group N & 6.00 & 1.50 & .75 \\
Group S & 3.38 & 2.00 & .63 \\
\hline
\end{tabular}

Table 3

Mean Number of Lines Crossed for Each Group During Each 10-Min Test Period

\begin{tabular}{lrrr}
\hline & Day 1 & Day 2 & \multicolumn{1}{c}{ Day 3 } \\
\hline Group H & 86.25 & 103.13 & 137.13 \\
Group N & 39.63 & 37.00 & 24.25 \\
Group S & 27.00 & 24.75 & 30.50 \\
\hline
\end{tabular}

\section{Behavioral Consequences}

A substantial correlation existed between the means and the standard deviations of the three groups for the durations of restraint-induced immobility, and, as might be expected, tests for homogeneity of variance (Winer, 1971) indicated that the raw data did not support this assumption for use of parametric analysis of variance. Both square-root and logarithmic transformations of the data were performed, but neither of these manipulations eliminated the heterogeneity of variance. For this reason, a Kruskal-Wallis test was applied to these data (Siegel, 1956). A significant effect was found $(\mathrm{H}=9.79$, $\mathrm{df}=2, \mathrm{p}<.01)$. The median and range for the duration of immobility for each group are presented in Table 1. Mann-Whitney $U$ tests indicated that Group $\mathrm{H}$ differed significantly from the other groups, which did not differ from each other.

In addition to the differences in median duration, the incidence of restraint-induced immobility also differed among the groups. Seven of the eight rats with hippocampal lesions demonstrated immobility. Only four of the eight unoperated rats and four of the eight sham-operated rats demonstrated immobility. The longest duration of immobility exhibited by a control rat was $45 \mathrm{sec}$, while five of the rats with hippocampal lesions exhibited durations of $39 \mathrm{sec}$ or longer. The lesion, therefore, dramatically increased both incidence and duration of restraint-induced immobility.

The data for the number of shuttlebox center crossings were found to satisfy the assumption of homo- geneity of variance and were subjected to repeated measures analyses of variance (ANOVA; Winer, 1971). No significant effects were found for the number of crossings either before or after shock. Table 2 presents the mean number of crossings in response to shock for each group on each test day. A significant group effect was found $(\mathrm{F}=11.55$, $\mathrm{df}=2,21$, $\mathrm{p}<.001)$, as was a significant days effect $(\mathrm{F}=22.46$, df $=2,42, p<.001$ ), but the Group by Days interaction was not significant. Subsequent Newman-Keuls tests indicated that Group $\mathrm{H}$ differed from both control groups, which did not differ from each other.

The data collected from the rats while they were tested in the larger arena also met the assumption of homogeneity of variance and were analyzed with a repeated measures ANOVA. The mean number of lines crossed by each group during each test period is presented in Table 3. A significant group effect was obtained for these data $(F=17.91$, $d f=2,21$, $\mathrm{p}<.001)$. Subsequent Newman-Keuls tests indicated that Group $\mathrm{H}$ differed from both control groups, which did not differ from each other. Neither the days effect nor the interaction achieved statistical significance. No significant effects were found when the number of rearings and number of fecal pellets were subjected to ANOVAs.

\section{DISCUSSION}

In the present experiment, bilateral electrolytic lesions of the hippocampus were found to increase the duration of restraint-induced immobility in the rat. This result replicates, in the rat, the observation that bilateral hippocampal destruction increases this form of immobility in the rabbit (Woodruff et al., 1975) and indicates that this effect of hippocampal destruction is not restricted to the rabbit. Compared to the control groups, the rats with hippocampal lesions also demonstrated increased activity in an open field and in response to footshock. These findings replicate previous findings reported by other authors (Blanchard et al., 1977; Isaacson, 1974) and demonstrate that our lesions produced behavioral effects equivalent to effects previously reported.

The relative insusceptibility to restraint-induced immobility demonstrated by the rats in the control groups is consistent with previous reports (Klemm, 1971; Ratner, 1967). Restraint-induced immobility can be obtained quite readily in rat pups tested between Postnatal Days 10 and 25 (McGraw \& Klemm, 1969; Svorad, 1957). Because the apical dendrites of the neocortical pyramids complete development at about the same time that rats become insusceptible to restraint-induced immobility, Klemm (1971) has argued that the neocortex inhibits this form of immobility in the adult rat. Further support for this hypothesis comes from experiments by McGraw and 
Klemm (1969) in which neodecorticate adult rats exhibited restraint-induced immobility, and by Teschke, Maser, and Gallup (1975) in which neocortical spreading depression was found to enhance restraint-induced immobility in mature rats.

However, both the time course of development and the effects of lesions produced in adult animals implicate the hippocampus in inhibition of restraintinduced immobility. Altman (1967) has reported that the dentate region of the hippocampal formation does not complete development until about Postnatal Day 25. The results of the present experiment indicate that electrolytic lesions of the hippocampus increase restraint-induced immobility in the adult rat, and Woodruff et al. (1975) demonstrated that aspiration lesions of the hippocampus in the rabbit significantly increase the duration of restraint-induced immobility in that species.

In our Fink-Heimer preparations, some degeneration was found in the corpus callosum and it might be argued that the enhancement of restraint-induced immobility produced by the lesions in the present study was due to inadvertent undercutting of the neocortex. However, complete analysis of the patterns of degeneration produced by these lesions did not support this contention. Degeneration was observed in areas that others (e.g., Valenstein \& Nauta, 1959) have reported to contain degeneration subsequent to lesions of the hippocampus, but degeneration attributable to destruction of neocortical efferents was not observed in such structures as the internal capsule. Moreover, inspection of the thionin- and Weil-stained sections did not reveal any widespread destruction of either the neocortical cell layers or underlying fiber systems.

All of these observations support the contention that the intact hippocampal formation inhibits other areas, presumably located in the reticular formation (Klemm, 1971), responsible for production of restraint-induced immobility. In addition, while our results are not compatible with the proposition that hippocampal lesions always result in a decreased tendency to become immobile in aversive situations, they are congruent with the hypothesis developed by Altman et al. (1973) that, in the adult rat, the hippocampus inhibits behaviors which are exhibited by neonatal or "juvenile" rats.

\section{REFERENCES}

Altman, J. Postnatal growth and differentiation of the mammalian brain, with implications for a morphological theory of memory. In G. D. Quarton, T. Melnechuk, \& F. O. Schmitt (Eds.), The neurosciences: $A$ study program. New York: Rockefeller University Press, 1967.

Altman, J., Brunner, R. L., \& Bayer, S. A. The hippocampus and behavioral maturation. Behavioral Biology, 1973, 8, 557596.

BlanchaRd, R. J., \& BlanchaRd, D. C. Effects of hippocampal lesions on the rat's reaction to a cat. Journal of Comparative and Physiological Psychology, 1972, 78, 77-82.
Blanchard, D. C., Blanchard, R. J., Lee, E. M. C., \& Fukunaga, K. K. Movement arrest and the hippocampus. Physiological Psychology, 1977, 5, 331-335.

Blanchard, R. J., \& Fial, R. A. Effects of limbic lesions on passive avoidance and reactivity to shock. Journal of Comparative and Physiological Psychology, 1968, 66, 606-612.

CARLI, G. Animal hypnosis in the rabbit. The Psychological Record, 1977, 27, 123-143.

Clark, G. Staining procedures. Baltimore: Williams \& Wilkins, 1973.

Gallup, G. G., JR. Animal hypnosis: Factual status of a fictional concept. Psychological Bulletin, 1974, 81, 836-853.

IsAaCson, R. L. The limbic system. New York: Plenum, 1974.

Isaacson, R. L., Douglas, R. S., \& Moore, R. Y. The effect of radical hippocampal ablation on acquisition of avoidance response. Journal of Comparative and Physiological Psychology, 1961, 54, 625-628.

IsaACson, R. L., \& Wickelgren, W. O. Hippocampal ablation and passive avoidance. Science, 1962, 138, 1104-1106.

KIM, C., KIM, C. C., KIM, J. K., KIM, M. S., ChANG, H. K., KIM, J. Y., LEE, I. G. Fear response and aggressive behavior of hippocampectomized house rats. Brain Research, 1971, 29, 237-251.

KLEMM, W. R. Neurophysiologic studies of the immobility reflex (“animal hypnosis"). In S. Ehrenpreis \& O. C. Solnitzky (Eds.), Neurosciences research (Vol. 4). New York: Academic Press, 1971.

McGraw, C. P., \& KLEmm, W. R. Mechanisms of the immobility reflex ("animal hypnosis"):III. Neocortical inhibition in rats. Communications in Behavioral Biology, 1969, 3, 53-59.

Papsdorf, J. D., \& WoodrufF, M. L. Effects of bilateral hippocampectomy on the rabbit's acquisition of shuttlebox and passive avoidance responses. Journal of Comparative and Physiological Psychology, 1970, 73, 486-489.

Pellegrino, L. J., \& Cushman, A. J. A stereotaxic atlas of the rat brain. New York: Appleton-Century-Crofts, 1967.

RATNER, S. C. Comparative aspects of hypnosis. In J. E. Gordon (Ed.), Handbook of clinical and experimental hypnosis. New York: Macmillan, 1967.

Siegel, S. Nonparametric Statistics. New York: McGraw-Hill, 1956.

Siegel, A., Edinger, H., \& Ohgami, S. The topographical organization of the hippocampal projection to the septal area: A comparative neuroanatomical analysis in the gerbil, rat, rabbit, and cat. Journal of Comparative Neurology, 1974, 157, 359-378.

Svorad, D. "Animal hypnosis" (Totstell reflex) as an experimental model for psychiatry. AMA Archives of Neurology and Psychiatry, 1957, 77, 533-539.

Teschke, E. J., Maser, J. D., \& Gallup, G. G., Jr. Cortical involvement in tonic immobility ("animal hypnosis"): Effect of cortical spreading depression. Behavioral Biology, 1975, 13, 139-143.

Valenstein, E. S., \& Nauta, W. J. H. A comparison of the distribution of the fornix system in the rat, guinea pig, cat, and monkey. Journal of Comparative Neurology, 1959, 113, 337-363.

Wirtanen, J. T. Selective silver impregnation of degenerating axons and axon terminals in the central nervous system of the monkey (Macaca mulatta). Brain Research, 1969, 14, 546-548.

Wine R, B. J. Statistical principles in experimental design. New York: McGraw-Hill, 1971.

Woodruff, M. L., Hatton, D. C., \& Meyer, M. E. Hippocampal ablation prolongs immobility response in rabbits (Oryctolagus cuniculus). Journal of Comparative and Physiological Psychology, 1975, 88, 329-334.

(Received for publication September 15, 1978; revision accepted May 15, 1979.) 